\title{
NUMERICAL INVESTIGATION OF LOAD SEQUENCE \\ EFFECT AND ENERGY DISSIPATION IN CONCRETE \\ DUE TO COMPRESSIVE FATIGUE LOADING USING THE NEW MICROPLANE FATIGUE MODEL MS1
}

\author{
M. AGUILAR*, A. BAKTHEER* AND R. CHUDOBA* \\ * Institute of Structural Concrete \\ RWTH Aachen University \\ Mies-van-der-Rohe-Straße 1, 52074 Aachen , Germany \\ e-mail: maguilar@imb.rwth-aachen.de, web page: http://www.imb.rwth-aachen.de/
}

\begin{abstract}
Modeling the fatigue behavior of concrete is a challenging task that has attracted the interest of researchers during the last decades. A new formulation of a microplane fatigue model denoted as MS1 introduced by the authors is employed. It aims to capture the basic inelastic mechanisms that are driving the tri-axial stress redistribution within a material zone during the fatigue damage process in concrete. In order to do so, the fatigue damage evolution is linked to a measure of the cumulative inelastic shear strain, reflecting the accumulation of fatigue damage owing to internal shear/sliding between aggregates at subcritical load levels.

In the first place, numerical studies are presented, in which the elementary interface behavior and dissipation mechanisms are evaluated. Later on, an evaluation of the energy dissipation of experimental tests in terms of their hysteretic loops is displayed. This approach is called into question as available experimental data strongly suggests that this method underestimates the actual amount of energy being dissipated. The microplane material model MS1, which is able to reproduce the concrete behavior under monotonic, cyclic, and fatigue loading with consistent sets of material parameters, has been employed for evaluating the contributions of the different dissipative mechanisms for different loading scenarios. Further studies assessing the ability of the model to reproduce the sequence effect for fatigue loading with varying amplitudes in terms of an energetic-based approach are presented.
\end{abstract}

